\title{
El capital en el siglo XXI de Thomas Piketty
}

DOI: https://doi.org/10.32870/dse.v0i11.280

\section{Juan Gerardo Martínez Borrayo* Citlalic López Aceves**}

Piketty, Thomas. El capital en el siglo XXI. México: Fondo de Cultura Económica. Sección de Obras de Economía, 2014, 663 pp.

Thomas Piketty es un economista francés que publicó en 2013 este libro que comienza a ser considerado un clásico de la economía y que, para sorpresa de muchos, es ya un best seller, algo inesperado para un libro de economía.

El libro es valioso ya que por un lado escribe de un tema que nos debería de importar a todos $\mathrm{y}$, por el otro, presenta su información de manera rigurosa pero clara; tiene la virtud de que es fácil de leer por cualquiera que no sea un especialista en el área.

Hay dos conclusiones generales que se deben destacar. La primera es que la historia de la distribución de la riqueza es una cuestión eminentemente política y no un tema solo de economía. Esto en parte está demostrado por el hecho de que en el siglo XX hubo dos periodos de reducción de la desigualdad que están ligados a las dos guerras mundiales y a las políticas públicas instrumentadas después de esas conflagraciones.

La segunda conclusión a la que llega es que hay mecanismos que reducen la desigualdad y los hay también que la aumentan. El principal mecanismo de reducción de la desigualdad es el proceso de difusión de los conocimientos, la inversión en la capacitación y formación de habilidades, además de las políticas seguidas en materia de educación, acceso a la formación, así como de instituciones creadas en ese campo.

Por otro lado, los mecanismos que aumentan la desigualdad son, en primer lugar un bajo crecimiento y, en segundo lugar, un elevado rendimiento del capital. Sobre todo este segundo, y lo resume en la fórmula .

* Doctor en Ciencia del Comportamiento con orientación en Neurociencias. Adscrito al Departamento de Neurociencias del Centro Universitario de Ciencias de la Salud, Universidad de Guadalajara. Correo electrónico: juan.gerardo.martinez.borrayo@, gmail.com

** Maestría en historia del mundo hispánico en el CSIS, Madrid España y maestrante en Desarrollo Social. Gestión en Responsabilidad Social Empresarial. Universidad Panamericana. Profesora de asignatura del Departamento de Humanidades de la Universidad Panamericana. Correo electrónico: cilopez@up.edu.mx 
Para explicar en qué consisten estos y otros mecanismos que generan desigualdad, Piketty nos habla en el primer capítulo sobre qué es el ingreso nacional (el conjunto de los ingresos de los que disponen los residentes de un país a lo largo de un año), qué es el capital (es el valor total estimado a los precios de mercado de todo lo que poseen los residentes y el gobierno de un países dado, en un momento determinado; siempre y cuando pueda ser intercambiado en un mercado) y cuál es la relación capital/ingreso (la cantidad total de riquezas poseídas en un tiempo dado, puede ser comparada con la cantidad de riquezas producidas y distribuidas en un periodo dado); esta relación se vincula con la participación de los ingresos del capital en el ingreso nacional dando como resultado la primera ley fundamental del capitalismo: , en donde $\alpha$ es la participación de los ingresos del capital en el ingreso nacional, $r$ es la tasa de rendimiento promedio del capital y $\beta$ es la relación capital/ ingreso. Con el fin de ilustrar esta ley el resto del capítulo lo dedica a la descripción de las grandes líneas de evolución de la distribución mundial del ingreso y la producción.

En el segundo capítulo analiza con detenimiento la evolución de las tasas de crecimiento de la población y de la producción después de la revolución industrial. La relación entre ambas es simple de entender, si el crecimiento de un país es de $3 \%$ y su población crece $1 \%$, entonces la tasa de crecimiento es en realidad de $2 \%$. A lo largo de prácticamente toda la historia de la humanidad tales cuentas dan como resultado un crecimiento de 0 . Pero es a partir del año 1700 que la producción realmente ha crecido a pesar de que también ha habido un aumento de la población. Esto es aún más cierto para el último siglo.

Tomado en su conjunto los últimos trescientos años, el crecimiento de toda la humanidad ha sido de $.8 \%$. Esta cantidad puede sonar ridícula (todo el mundo quiere crecer a tasas de $7 \%$ o más, Fox dixit), pero en realidad es un crecimiento sumamente rápido; esta idea es conocida como la ley del crecimiento acumulado (una tasa de rendimiento anual de algunos puntos porcentuales, acumulada sobre varias décadas, lleva mecánicamente a un muy significativo incremento del capital).

El problema es que a futuro se esperan dos cosas, una es que la tasa de crecimiento demográfico en todo el mundo está bajando de tal manera que, para el año 2100, se cree que va a ser la misma que existía hace mil años. Y, en segundo lugar, Piketty augura que para la misma fecha, también la tasa de crecimiento va a bajar probablemente a menos de la mitad de la que existe en la actualidad.

Los siguientes cuatro capítulos constituyen la segunda parte del libro llamada "la dinámica de la relación capital/ingreso". Lo que trata de hacer en esta parte es analizar la manera en que se presenta, en el inicio del siglo XXI, el tema de la evolución a largo plazo de la relación capital/ingreso y del reparto global del ingreso nacional, entre ingresos por trabajo e ingresos por capital.

El capítulo III presenta los cambios del capital desde el siglo XVIII en el Reino Unido y Francia. En estos dos países básicamente el capital nacional (medido como \% del ingreso nacional) ha pasado de ser eminentemente agrícola a capital inmobiliario, industrial y financiero. Pero lo más interesante es que si se hace la división del capital privado y del público, se observa que desde 1700 hasta 1910, en ambos países, el capital privado es mayoritario; pero de 1910 a 1970 hay una 
drástica reducción de ese capital. Y lo mismo pasa para los casos de Alemania y de Estados Unidos, que es lo que analiza el capítulo IV.

Una de las cosas que llama la atención de lo que presenta en los capítulos III y IV es que a pesar de los cambios en la naturaleza del capital, su valor no cambió o bien, cuando lo hizo, tendió a volver a los mismos niveles históricos. Su respuesta es la segunda ley fundamental del capitalismo. La conocida relación entre capital e ingreso $\beta$ la vincula con la tasa de ahorro del país $s$ y su tasa de crecimiento de su ingreso nacional $g$ y la fórmula es la siguiente $\beta=s / g$. Esto es lo principal que analiza en el capítulo V. Utilizando el mismo ejemplo que presenta Piketty, si suponemos que un país ahorra $12 \%$ de su ingreso y si la tasa de crecimiento es $2 \%$ anual, entonces el país en cuestión tenderá a acumular $600 \%$ del ingreso nacional en capital. Esta es una ley válida sólo a largo plazo, se necesitan décadas para que se cumpla la ley. En este momento, la relación capital/ingreso en el mundo se acerca a 500\% y especula que para finales del siglo será de $700 \%$.

La siguiente pregunta que trata de responder Piketty en el capítulo VI es cuánto del ingreso es debido al capital y cuanto al trabajo. Su análisis histórico muestra que en términos generales la mayor parte de los ingresos provienen del capital y no del trabajo (70 y $30 \%$ respectivamente), esto a pesar de que el rendimiento del capital ha sido 4 o $5 \%$ en los siglos XVIII y XIX, y 3 y $4 \%$ hoy en día. Si recordamos que hay una perspectiva de crecimiento bajo y un incremento demográfico nulo, entonces los patrimonios provenientes del pasado adquieren cada vez más una mayor importancia.

Al ingresar a la tercera sección del libro, titulada "La estructura de la desigualdad", comienza a tratar el tema que en realidad más le interesa: cómo se está concentrando la riqueza a nivel individual. La pregunta central que se plantea en el capítulo VII es: ¿qué es más importante en este fenómeno de concentración: el trabajo o la herencia? Su respuesta es que, a pesar de la muy difundida creencia de que las desigualdades se basan en el trabajo y el mérito individual, en realidad el capital siempre está distribuido de manera más desigual que el trabajo. Como hablar de más o menos desigualdad es un tanto ambiguo, introduce unas tablas en las que específica que una desigualdad baja es aquella en la que $10 \%$ de los más ricos tienen $25 \%$ de los ingresos, mientras que desigualdad muy elevada va a ser aquella en la que ese mismo grupo de personas tenga $60 \%$. Si recordamos que el capítulo VI nos dice que la mayor parte de los ingresos vienen del capital, entonces la concentración está más ligada a los ingresos provenientes del capital que del trabajo; y el capital es una cuestión más de herencia que de méritos propios.

Llegamos así al capítulo VIII, titulado "Los dos mundos", que es probablemente el más revelador de todos. Como muestra del fenómeno mundial que se está presentando de concentración de la riqueza, describe el caso de Francia. En 1932, 5\% de la población más rica era la que recibía más ingreso por el capital que por el trabajo; esa cifra se redujo en 2005 a .1\%; es decir, mucho menos gente se estaba haciendo realmente rica. Este fenómeno se inició desde la década de 1980 en Francia y en la de 1970 en los Estados Unidos, con la diferencia de que en este último país la concentración de la riqueza es mucho más acusada. 
Que la mayor parte de los ingresos sean provenientes del capital no quiere decir que el trabajo no sea una fuente de riqueza, sobre todo si recordamos que la capacitación para el trabajo, es decir la educación, es la principal forma de distribución de la riqueza. Pero resulta que la concentración de la riqueza se explica también por la aparición de lo que Piketty llama los "superejecutivos", que son aquellos sujetos que ganan enormes cantidades de dinero por su trabajo (son parte del percentil superior de ricos). Esto es sobre todo cierto para los países anglosajones (Estados Unidos, Reino Unido, Canadá y Australia), menos para Europa y Japón y, al parecer, aún más dramático para los países emergentes (presenta datos de la India, Indonesia, China, Sudáfrica, Argentina y Colombia). Todo esto es parte del capítulo IX.

En el capítulo X plantea como eje de análisis el siguiente punto: ¿cómo y por qué se dio la concentración de la riqueza que estamos observando? La respuesta la dimos en la primera fórmula que presentamos en este texto: . Es decir, ha habido una tasa de rendimiento del capital superior al crecimiento. De hecho siempre es así, pero, ¿por qué el rendimiento del capital es superior a la tasa de crecimiento siempre? Hay tres respuestas a esta cuestión: tiempo, crecimiento e impuestos. Pero lo principal son los impuestos: los impuestos al capital son muy reducidos. Si el siglo XX se caracterizó por una baja en la desigualdad, pero a partir de los años ochenta ésta ha ido aumentando, su conclusión es que el siglo XXI va a ser más desigual que el XX.

El capítulo XI analiza a profundidad el papel que jugarán a largo plazo la herencia y el mérito. El autor prevé que para el siglo XXI la herencia volverá a tener un papel preponderante en la acumulación de la riqueza, pues en el presente la tasa de rendimiento es mucho más alta que la tasa de crecimiento; además de mostrarse una disminución demográfica importante a futuro. Para el autor, el factor demográfico es determinante para el aumento del crecimiento económico, a menor población mayor acumulación de riqueza a largo tiempo - patrimonio de herencia - y por lo tanto mayor estancamiento en el crecimiento y en el aumento de la desigualdad, pues el capital no circula a los deciles inferiores que no cuentan con herencia.

En el capítulo XII, llamado "La desigualdad mundial en la riqueza en el siglo XXI", se pregunta si va a incrementarse de manera ilimitada en el siglo XXI el porcentaje del capital mundial propiedad de los ultraricos que aparecen en las clasificaciones de las revistas. Piketty cree que no va a ser así, porque eso llevaría a un colapso de la economía ya que el ahorro de todo el dinero que no puede gastarse ya no se sabe en dónde invertirse y por ello el rendimiento mundial del capital acabará por desplomarse.

Y entramos así a la última parte del libro, a la que Piketty llama "Regular el capital en el siglo XXI”. ¿Cuál es la propuesta del autor para contrarrestar los efectos de la desigualdad entre la tasa de rendimiento frente a la tasa de crecimiento? Los nombres de los capítulos que constituyen esta sección dan la respuesta. El capítulo XIII se llama "Un estado social para el siglo XXI"; el XIV, "Repensar el impuesto progresivo sobre el ingreso"; el XV, "Un impuesto mundial sobre el capital" y el XVI, "La deuda pública". 
Sobre el estado social dice que esto significa financiar la educación, salud y jubilaciones. Basándose en John Rawls y Amartya Sen, propone que sea la formación de capacidades, vía una educación competente e incluyente para todos, una de las vías para combatir la desigualdad.

El otro punto central de su propuesta son los impuestos. Se debe organizar con eficacia el financiamiento público y por ello debe estructurarse el sistema fiscal para que entre más riqueza posean las personas más impuestos paguen.

La propuesta de mayor trascendencia del autor se refiere a la formación de un impuesto mundial sobre el capital. ¿A qué se refiere el autor con esto? La idea es formar un impuesto de manera progresiva y lenta, primero regionalmente, luego de manera nacional, continental y después mundial sobre el capital individual, es decir, el sobre el valor neto de los activos que cada persona controla.

El impuesto mundial sobre el capital es considerado por Piketty como necesario para evitar la creciente desigualdad vaticinada para el siglo XXI, además de ser complementario de los impuestos progresivos en ingreso, herencia y capital. Sin embargo, antes de imponer el impuesto mundial sobre el capital es necesario democratizar y transparentar la riqueza que poseen unos y otros en el ámbito internacional, y buscar un mecanismo de transparencia financiera internacional.

Por último, el debate en muchos países ha sido cómo reducir la deuda pública. Generalmente se ha propuesto que se puede reducir con medidas impositivas, inflacionarias o de austeridad. Pero, en su opinión el debate debería de ser cómo reducir la desigualdad y eso sólo es posible con la participación del Estado en la economía, por medio de reformas al sistema fiscal y su democratización en la información, transparencia sobre las grandes fortunas para evitar paraísos fiscales; asimismo, aumentar los impuestos progresivos sobre el capital y la herencia, concentrándose principalmente en las altas fortunas, para de esta manera distribuir el capital a los demás deciles, complementado a su vez con la formación de un impuesto mundial sobre el capital. 